\title{
Endopróteses: opção terapêutica para estenoses traqueobrônquicas adquiridas
}

\section{Tracheobronchial stents: terapeutic option for acquired tracheobronchial stenosis}

\author{
Daniel Marchi dos Anjos', Jader Joel Machado Junqueira', \\ Diogo Bugano Diniz Gomes², Ricardo Mingarini Terra ${ }^{3}$, \\ Hélio Minamoto ${ }^{3}$, Fabio Biscegli Jatene ${ }^{4}$
}

\begin{abstract}
Anjos DM dos, Junqueira JJM, Gomes DBD, Terra RM, Minamoto H, Jatene FB. Endopróteses: opção terapêutica para estenoses traqueobrônquicas adquiridas. Rev Med (São Paulo). 2007 jul.-set.;86(3):174-84.

RESUMO: A estenose traqueobrônquica é uma condição que cursa com dispnéia, estridor e pneumonia obstrutiva, causada por diversas condições como tumores, compressão extrínseca e traqueobroncomalacia, porém a causa mais freqüente é a complicação resultante de intubação prolongada, sendo que ocorre em aproximadamente $1 \%$ das intubações. O tratamento de escolha para esta condição é a ressecção cirúrgica do segmento atingido, entretanto este procedimento não é possível em todos os pacientes e isto levou à adoção de técnicas endoscópicas paliativas. O uso de endopróteses para manter uma via aérea pérvia está entre as técnicas endoscópicas com melhores resultados. As endopróteses utilizadas atualmente dividem-se, principalmente, em próteses de silicone e metálicas expansíveis, cada uma com vantagens e desvantagens específicas. A presente monografia visa apresentar os principais modelos de endopróteses, comparando suas vantagens e desvantagens e índices de complicações, procurando sistematizar as indicações ideais para cada tipo de prótese.
\end{abstract}

DESCRITORES: Constrição patológica. Traquéia/cirurgia. Implante de prótese. Dispnéia/ etiologia.

\footnotetext{
1. Acadêmico do 5을 Ano do Curso de Medicina da Faculdade de Medicina da Universidade de São Paulo (FMUSP).

2. Acadêmico do $4^{\circ}$ Ano do Curso de Medicina da Faculdade de Medicina da Universidade de São Paulo (FMUSP).

3. Médico Assistente do Serviço de Cirurgia Torácica do Hospital das Clínicas da FMUSP (HCFMUSP) - Orientador.

4. Diretor do Serviço de Cirurgia Torácica do HCFMUSP - Orientador.

Endereço para correspondência: Laboratório Anátomo-Cirúrgico do Instituto do Coração (InCor) - HCFMUSP. R. Dr. Enéas de Carvalho Aguiar, 44 - 2ํA Andar / Bloco 1 - Cerqueira César. CEP: 05403-900 - São Paulo/SP. E-mail: jader_junqueira@yahoo.com.br
} 


\section{INTRODUÇÃO}

A estenose traqueobrônquica, caracterizada por uma redução da luz das vias áreas superiores, é uma condição que cursa com dispnéia, estridor e pneumonia obstrutiva. Dependendo da localização e da extensão comprometida uma pequena exacerbação da obstrução (causada por edema, acúmulo de secreções ou sangue) pode significar risco imediato de vida.

Apesar de existirem estenoses traqueais congênitas, idiopáticas, aquelas resultantes de doenças auto-imunes e de neoplasias, a causa principal (apesar de sua incidência vir diminuindo ultimamente) é a decorrente de complicação de intubação prolongada ${ }^{1,2}$. Nestes casos, devido a uma excessiva pressão na traquéia pelo cuff dos tubos endotraqueais, ocorre necrose isquêmica local da cartilagem traqueal e excessiva proliferação de tecido de granulação. Os fatores de risco conhecidos para esta complicação são intubação durante mais de 10 dias, pressão do cuff do tubo endotraqueal maior do que $30 \mathrm{mmHg}$, mobilidade excessiva do tubo, infecção concomitante, tubos calibrosos e intubações traumáticas ou repetitivas ${ }^{3}$. Estenose benigna clinicamente significativa ocorre em aproximadamente 1 a $2 \%$ dos pacientes após intubação ${ }^{4,5}$.

Os objetivos do tratamento desta condição são, em ordem de prioridade: 1 - obter uma via aérea patente; 2 - preservar a função glótica para proteger a via contra aspiração e; 3 - manter uma qualidade da voz aceitável ${ }^{6}$. O tratamento de escolha para a estenose traqueal é a ressecção do segmento atingido com posterior anastomose término-terminal, devido à altas taxa de sucesso e excelentes resultados, especialmente a longo prazo, além de pequenas taxas de complicações e de reincidência ${ }^{7,8,9}$. De fato, a experiência reportada pelo serviço de cirurgia torácica pela escola de medicina da Harvard, demonstra resultados bons ou excelentes em $94 \%$ de mais de 500 ressecções $^{10}$. Entretanto, em alguns casos este procedimento é impossível (por exemplo em longas estenoses, tentativa anterior de reconstrução cirúrgica sem sucesso, tumores metastáticos ou irressecáveis, recusa do paciente) o que demanda tratamento nãocirúrgico ${ }^{7,11}$.

Para estas situações, desenvolveram-se técnicas endoscópicas paliativas que, apesar do resultado a longo prazo ser freqüentemente ruim, a desobstrução permanente e/ou temporária da via aérea produz significativa melhora da qualidade de vida e um potencial aumento na expectativa de vida.

Entre estas técnicas paliativas minimamente invasivas, podemos citar a dilatação, a cirurgia a laser e o uso de endopróteses traqueais. Com raras exceções, as duas primeiras opções, isoladamente, produzem resultados pouco duradouros, sendo necessárias diversas intervenções ${ }^{12,13}$. $O$ uso de endopróteses traqueais produz resultados mais duradouros e, por isso, elas têm sido largamente empregadas em estenoses malignas e benignas causadas por compressão extrínseca, doença intraluminal, ou por perda do suporte cartilaginoso. Quando bem indicada e conduzida, esta técnica produz bons resultados em 80 a $95 \%$ dos pacientes ${ }^{14,15}$.

O conceito da utilização das próteses endotraqueais para se obter uma via aérea pérvia, e, assim, possibilitar ao paciente continuar respirando pelas vias aéreas superiores, preservando uma série de funções inerentes a elas (fonação, olfato e umidificação do ar inspirado) data do século XIX, quando Trendelemburg descreveu uma prótese metálica em forma de $\mathrm{T}$, que podia ter seu orifício externo fechado permitindo que o paciente respirasse normalmente. $\mathrm{A}$ primeira tentativa de colocação de endoprótese traqueobrônquica ocorreu em 1915, quando Brunings e Albrecht desenvolveram uma feita de borracha ${ }^{16}$.

Depois de anos de desenvolvimento de materiais e técnicas, Dumon, em 1990, apresentou a primeira endoprótese distribuída comercialmente feita de silicone ${ }^{17}$. Durante a década de 90 , avanços na tecnologia possibilitaram o desenvolvimento de diversas próteses feitas com variados materiais, de metal ao nitinol; da tecnologia primariamente usada em cirurgia vascular, resultou o desenvolvimento de endopróteses auto-expansíveis. Wallace ${ }^{18}$, em 1986, publicou os 2 primeiros casos de colocação com sucesso deste tipo de dispositivo.

Atualmente os esforços dirigem-se para 0 desenvolvimento de próteses bio-compatíveis que reduzissem as respostas imunológicas e de próteses degradáveis, que não necessitem de futuras intervenções para a sua retirada. Uma dessas novas promessas é a prótese feita de poly-l-lactide, um material absorvível que apresentou bons resultados em animais, mas que ainda não foi testado em humanos ${ }^{19}$. As indicações atuais ${ }^{20}$ para o uso de endopróteses traqueais estão discriminadas na Tabela 1. 
Tabela 1. Indicações para o uso de endopróteses traqueais

\begin{tabular}{cc}
\hline Benignas & Malignas \\
\hline - Carcinoma broncogênico & $\bullet$ Estenose pós-intubação \\
Lesões Intraluminais & - Inflamatórias \\
Lesões Extraluminais & Tuberculose \\
$\bullet$ Tumor primário de vias aéreas & Histoplasmose \\
Carcinoma de células escamosas & - Anastomóticas \\
Carcinoma adenóide cístico & Transplante de pulmão \\
Diversos & Ressecção em manga \\
- Lesões malignas extraluminais & $\bullet$ Traqueobroncomalacia \\
Câncer esofágico & $\bullet$ Estenose congênita \\
• Câncer de cabeça e pescoço & $\bullet$ Compressão por endoprótese esofágica \\
Câncer de Tireóide & \\
- Metástases de vias aérea & \\
Carcinoma de células renais & \\
Câncer de cólon &
\end{tabular}

Tipos de endoprótese

- Endopróteses de silicone rígido

\section{- Endoprótese de Montgomery}

Em 1965, Willian Montgomery ${ }^{21}$ desenvolveu uma endoprótese de silicone composta por dois tubos ocos que se interseccionam em forma de "T". A coluna vertical permanece na taqueia e deve atravessar toda a extensão da estenose, enquanto que o braço lateral se projetara pelo buraco de uma traqueostomia. Em uma modificação do desenho original, algumas possuem o braço lateral com ranhuras e um anel de silicone que deve ser preso justo à pele para evitar migração. Ela também possui uma "tampa" para impedir o fluxo de ar e permitir a fonação e a respiração nasal (em crises de dispnéia, essa oclusão deve ser removida e o paciente volta respirar pela traqueostomia). Existem diversas numerações da endoprótese que correspondem ao seu diâmetro externo e que variam de 6 a $16 \mathrm{~mm}$. O comprimento apresenta-se em tamanho pediátrico, padrão, longo e extralongo. Após medição broncoscópica da estenose, o tubo deve ser seccionado no tamanho exato com o bisturi e deve-se atentar para as pontas, que devem ser "suavizadas" com lixas para evitar lesões à parede traqueal.

Preferencialmente, a colocação da prótese deve ser feita com o paciente sob anestesia geral e com respiração espontânea. Uma broncoscopia rígida inical é feita a fim de se medir o comprimento da estenose e para realizar uma dilatação inicial, retirando-se possíveis tecidos de granulação. Se necessário, uma dilatação adicional pode ser feita usando velas de Hegar.

A técnica descrita por Montgomery consiste em, inicialmente, prender a porção distal da endoprótese com uma pinça de Kelly para deixar o tubo em forma de "U", reduzindo o seu diâmetro e facilitando a inserção pelo estroma traqueal. Uma segunda Kelly deve ser presa ao braço lateral por segurança. Em seguida, após a inserção da parte distal, empurra-se a endoprótese para baixo e insere-se a parte proximal (seguindo a mesma técnica) que então se estende para cima ${ }^{22}$.

Em casos em que a colocação da parte proximal do tubo é difícil, uma técnica descrita por Cooper et al. ${ }^{23}$ pode ser utilizada. Ela consiste em passar uma fita de traqueostomia pelo braço lateral e fazêla penetrar no estroma traqueal. Uma pinça inserida pelo broncoscópio rígido deve puxar a fita fazendo-a sair pela boca. Uma Kelly deve ser presa fortemente no braço lateral prendendo a fita. A seguir, insere-se a parte distal do tubo empurrando-o para baixo e puxa-se a fita que, então, serve de guia e facilita a entrada da parte proximal.

As pontas do tubo devem ultrapassar totalmente a extensão da lesão, pois este é um local potencial para formação de granulomatose. A parte proximal da endoprótese nunca deve ficar sobre a laringe subglótica, para evitar a formação de um anel que pode levar ao crescimento de granuloma. Em sua parte distal, o tubo deve atravessar toda a extensão da estenose, contudo não deve ser demasiadamente longo para que a eliminação de secreções não seja dificultada e nem que se oblitere ou irrite a carina, o que também pode levar à formação de granulomas. 
A revisão final da posição do tubo deve ser feita com um broncoscópio flexível e o teste de sua efetividade é realizado inserindo-se o tubo de ventilação pelo tubo e observando a saturação de oxigênio ${ }^{22}$.

\section{- Endoprótese de Dumon}

Em 1980, Dumon ${ }^{17}$ realizou experiências com uma endoprótese de silicone rígido em forma de " $T$ " similar à de Montgomery, porém com uma modificação simples e ao mesmo tempo fundamental: ao longo de todo o tubo longitudinal, existiam protuberâncias que a fixavam à parede da laringe e assim reduziam a incidência de uma das principais complicações de seu antecessor: a migração da prótese. A endoprótese de Dumon existe em dois modelos: Um em forma de "T" e o outro não possui um braço lateral e é utilizado em situações onde não exista ou não se exige uma traqueostomia.

A técnica para a colocação deste dispositivo é essencialmente igual à de Montgomery. Uma nova técnica ${ }^{24}$ descreve o uso de um tubo endotraqueal para a colocação da endoprótese. Inicialmente, com o paciente sob anestesia geral e respiração espontânea, a estenose é dilatada com o uso de ogivas metálicas ou laser. A seguir, sob observação broncoscópica e uso de fluoroscopia, um tubo endotraqueal de tamanho apropriado é inserido até após a extremidade distal da estenose. Então, a endoprótese é dobrada, inserida no tubo e empurrada até o sitio da lesão com o auxílio de um cabo de metal de aço inox com ponta arredondada. Esse cabo mantém a endoprótese no local enquanto o tubo é lentamente puxado para que a endoprótese, que estava dobrada, se expanda e fixe no lugar certo, o que é confirmado com visualização broncoscópica.

Esta técnica possui as seguintes vantagens sobre a convencional, que utiliza broncoscópio rígido: 1 - A introdução de tubo endotraqueal e broncoscópio flexível é mais fácil para o médico e menos estressante para o paciente; 2 - Apesar do diâmetro do introdutor flexível ser maior do que o do rígido original, sua introdução é mais fácil devido à sua flexibilidade; 3 - O procedimento é mais fácil e menos estressante para pacientes cuja broncoscopia rígida seria complicada (como pacientes que não conseguem estender o pescoço ou que o tumor tenha provocado um deslocamento traqueobrônquico). Entretanto, a fragilidade da preensão da pinça endoscópica flexível faz com que a retirada ou movimentação da endoprótese seja difícil, sendo mais indicado para isso a broncoscopia rígida ou traqueostomia. Essa nova técnica, portanto, é especialmente útil em estenoses malignas que, geralmente, não precisam de retirada da prótese.

\section{- Próteses de metal autoexpansível \\ - Endoprótese de Nitinol (Ultraflex)}

As endopróteses de metal auto-expansíveis, de uma maneira geral, assemelham-se aos stents usados na cirurgia vascular: são cilindros de uma malha de metal que inicialmente encontram-se contraídos e que, ao atingirem o local correto e serem acionados, expandem-se, aumentando o diâmetro da via aérea.

A prótese Ultraflex é formada por um filamento único de um composto de alumínio e titânio chamado de nitinol disposto em forma de uma malha cilíndrica. Esse composto apresenta fenômeno de "memória" da sua forma [25]: em baixas temperaturas ele se apresenta contraído e, ao atingir temperaturas maiores, como a do corpo humano, ele se expande. Assim, este dispositivo automaticamente se expande ao deixar o cateter de colocação e chegar na via aérea, no local da estenose. Esta prótese existe na versão "descoberta" e "coberta", sendo a última revestida por uma camada de algum material sintético para evitar que tecido de granulação ou tumoral cresça através dela.

A técnica ${ }^{26}$ de colocação inicia-se com a anestesia geral do paciente com intubação orotraqueal. Quando uma saturação de oxigênio adequada é obtida, o tubo é removido e a partir daí é utilizada ventilação intermitente, observando-se o nível de saturação (o ideal é sempre mantê-lo acima de $90 \%$ ). A seguir, endoscópios de diversos tamanhos são inseridos para examinar a traquéia e o segmento estenosado.

Depois que o segmento comprometido é localizado, fios de metal são posicionados sobre a pele, com o auxílio de fluoroscopia, para servirem de marcadores, delimitando o começo e o fim da estenose, auxiliando na colocação da endoprótese sob fluoroscopia. Uma prótese de diâmetro e comprimento adequados é escolhida, baseando-se em uma tomografia computadorizada anterior. O diâmetro dela, quando totalmente expandida, deve ser 1 a $2 \mathrm{~mm}$ maior que o da traquéia normal para permitir uma fixação mais segura à parede traqueal.

Com a ajuda de um fio-guia, o cateter de colocação é inserido sob visualização fluoroscópica. $O$ cateter deve ser alinhado para que seu centro fique entre os fios marcadores colocados previamente. Ele, então, é removido e a prótese, liberada. Esta parte do processo exige especial atenção, pois a prótese é liberada da parte distal para a proximal, então ela só pode ser movimentada nesta direção para corrigir possíveis erros. A seguir, um balão dilatador é inserido para dilatar a prótese até seu tamanho adequado. Devido às suas propriedades auto-expansíveis, espera-se que ela se dilate completamente dentro de 24 horas. Por 
fim, a inserção da prótese é confirmada por broncoscopia rígida. Uma variação desta técnica permite o uso de broncoscópio flexível e, portanto, elimina-se a necessidade de anestesia geral. Rotineiramente, realiza-se uma radiografia ou tomografia computadorizada depois de 24 horas para se averiguar a posição final da prótese.

Recentemente, uma nova técnica ${ }^{27}$ para a colocação deste tipo de prótese foi descrita, sem a necessidade do uso da fluoroscópica. Nela, a intubação não é utilizada para evitar traumas e sangramento no local da estenose. Utiliza-se o laringoscópio após a indução anestésica e os dados endoscópicos relevantes (local da estenose, comprimento desta, etc.) são obtidos com o uso da óptica de Hopkins. A prótese é liberada em uma posição ligeiramente mais proximal do que a ideal e, após a expansão parcial, é empurrada com a ajuda do cateter de sucção. Isso é recomendado porque a ponta proximal é de difícil visualização, dificultando o posicionamento exato; e porque, caso este não seja ideal, puxar a prótese pode resultar em dano à mucosa. Para completar a expansão, uma pinça de braços longos é utilizada. Nenhum auxílio radiológico é necessário durante este processo.

Os autores desta técnica defendem que ela elimina a exposição à radiação, que a visualização direta é importante (pois a Ultraflex é pouco radiopaca) e que este procedimento reduz a chance de trauma, o que diminui as chances de sangramento intraoperatório e de ocorrerem complicações posteriores, como a formação de tecido de granulação.

\section{- Endoprótese de Gianturco}

Esta prótese foi desenvolvida na década de 80 e é formada por um espiral contínuo de um monofilamento de aço inox disposto em forma de um "ziguezague" de 5 a 10 camadas, formando um cilindro ${ }^{28}$. Para prevenir migração, ela possui "ganchos" em sua porção distal e proximal que permitem se ancorar na parede da traquéia. Devido às suas características semelhantes à prótese Ultraflex, a técnica de sua colocação é essencialmente a mesma (descrita anteriormente).

- Prótese de silicone auto-expansível (Polyflex)

- Endoprótese de Malha de Poliéster Revestida

A prótese Polyflex é uma endoprótese de malha de poliéster revestida de silicone que procura obter as vantagens de ambas as endopróteses de silicone rígido e de metal autoexpansível, evitando suas des- vantagens. Sua colocação ${ }^{29}$ ocorre com o paciente sob anestesia geral, sendo realizada laringoscopia de suspensão para obtenção da via aérea. A dilatação da via é feita com ogivas metálicas ou balão hidrostático e, após a escolha do tamanho ideal da endoprótese baseada em aspectos broncoscópicos e radiológicos, esta é posicionada com um introdutor especial fornecido pelo fabricante. A seguir, a sua posição é verificada endosco-picamente.

\section{- Próteses dinâmicas}

\section{- Protese Dinâmica (Rüsch)}

A prótese dinâmica desenvolvida por Rüsch ${ }^{30}$ consiste em um tubo de silicone em forma de "Y", com um tubo central simulando a traquéia, seguida de uma bifurcação com um prolongamento direito curto e um esquerdo longo simulando a anatomia da árvore brônquica. A parte anterior possui implantes de metal que se assemelham aos anéis incompletos da traquéia e a membrana posterior dessa prótese pode invaginar durante a tosse para facilitar o fluxo do muco.

Sua colocação inicia-se com uma avaliação inicial com broncoscópio rígido para avaliar a extensão da estenose e para adaptar o tamanho da endoprótese, cortando as terminações se necessário, bem como para escolher o calibre ideal. A seguir, utiliza-se o laringoscópio direto e os fórceps adequados para posicioná-la. O broncoscópio rígido é re-introduzido para se fazer a avaliação final da posição e do funcionamento da endoprótese ${ }^{30}$.

\section{Vantagens e desvantagens}

A endoprótese ideal é aquela que apresenta: 1 - fácil colocação e remoção; 2 - força de expansão suficiente para manter uma via aérea pérvia sem, contudo, causar dano à mucosa; 3 - diversos tamanhos para se adaptar à vias aéreas de diferentes calibres e à lesões de diferentes extensões; 4 - capacidade de permanecer no local em que foi posicionada, sem apresentar migração; 5 - material inerte que não irrita a mucosa, precipita infecções ou facilita a formação de tecido de granulação e; 6 - preservação da função do epitélio ciliar, mantendo a eliminação de muco e secreções ${ }^{31-34}$.

Atualmente, nenhuma endoprótese utilizada pode ser considerada ideal, todas possuem vantagens e desvantagens que a particularizam para diversas situações. $A$ isso, adiciona-se a impossibilidade de se analisar o real impacto dos diferentes tipos de endopróteses pela dificuldade de se produzir estudos randomizados para tal fim, pois estes esbarram em 
aspectos éticos (uma vez que a melhora obtida com as próteses é significativa) e na dificuldade de encontrar pacientes, que muitas vezes encontram-se dispnéicos, dispostos a aceitar somente a observação sendo que são conhecidas outras formas de tratamento ${ }^{35}$.

As próteses de silicone possuem as vantagen $\mathrm{s}^{22,36}$ de serem removíveis após a inserção (o que as qualificam para estenoses benignas), de produzirem pouca reação tecidual, de serem impermeáveis ao crescimento de tumor (por serem um cilindro fechado, em oposição à malha permeável das próteses de metal) e de serem mais baratas que as próteses de metal auto-expansível.

Suas principais desvantagens ${ }^{14,37}$ consistem em: possibilidade de migração da prótese, pois, diferentemente das metálicas expansíveis, estas endopróteses não apresentam ganchos para a fixação na parede interna das vias aéreas e sua força radial é, relativamente, menor. Na literatura ${ }^{38,39,40}$, a taxa deste tipo de complicação encontra-se em torno de $18 \%$; interferência no funcionamento do epitélio ciliar da traquéia embaixo da prótese, o que pode levar ao acúmulo de secreção no lúmen e obstrução da via aérea, sendo que esta complicação é observada em $16 \%$ dos $\operatorname{casos}^{38-41}$; uma menor proporção entre o diâmetro interno e externo da prótese se comparada com as de metal e necessidade do uso de broncoscopia rígida e de anestesia geral para a sua inserção. Wood et. al. ${ }^{42}$ publicou um importante estudo com 143 pacientes com próteses de silicone (na maioria - $71 \%$ - tubos simples de silicone, sendo que em $13 \%$ dos casos foram colocadas próteses metálicas expansíveis) onde os índices de complicações foram: $5 \%$ migração; $27 \%$ obstrução parcial por secreção; $9 \%$ obstrução parcial por tecido de granulação e $1 \%$ perfuração da via aérea.

Alguns avanços foram feitos em modelos mais recentes para evitar alguns desses problemas, como a adição de protuberâncias na parte externa na prótese de Dumon para evitar a migração e, nas próteses dinâmicas a parte posterior flexível facilita a eliminação de secreções.

As próteses de metal auto expansíveis possuem as vantagens ${ }^{22,26,43}$ de serem mais facilmente inseridas e fixadas; de apresentarem neoepitelização, onde a prótese é incorporada à parede traqueal, recuperandose a função do epitélio ciliar e a remoção do muco e das secreções; de praticamente não apresentarem migração; de se acomodarem a diversos diâmetros traqueais (pelo fato de serem auto-expansíveis, o que elimina a possibilidade de erro humano na hora da escolha do diâmetro da prótese) e de possuírem uma maior proporção entre os diâmetros externo e interno.
Suas desvantagens ${ }^{37,44}$ incluem a força radial que exercem contra a parede da via aérea, o que pode levar a erosão dessa parede e surgimento de fístulas; o fato de serem permeáveis ao crescimento de tumores e de tecido de granulação, o que pode levar à obstrução; a possibilidade de quebrarem, principalmente quando usadas durante muito tempo; e sua difícil remoção, que pode necessitar de cirurgia aberta.

Próteses metálicas apresentam uma maior taxa de crescimento de tecido de granulação do que as de silicone $^{32}$ por apresentarem uma maior rigidez, causando maior irritação, e por não apresentarem pontas terminais, como as de silicone, mas múltiplas pontas que exercem pressão sobre o epitélio favorecendo a irritação45. Em um grande estudo retrospectivo, Saad et. al. ${ }^{46}$ reportaram uma taxa de $14,6 \%$ de granulomas obstrutivos relacionados à colocação de 112 endoprótoses metálicas expansíveis (Wallstent e Ultraflex). Além de causar obstrução da via aérea, o tecido de granulação pode servir como um meio de cultura de bactérias, favorecendo infecções; culturas feitas com este tipo de tecido mostraram-se positivas para Streptococcus viridians, Pseudomonas aeruginosa e Staphylococcus aureus.

As razões para quebra deste tipo de endoprótese incluem a força a que são submetidas durante a expectoração ${ }^{44}$ e a deterioração em longo prazo ${ }^{44,47}$. Rousseau ${ }^{48}$ publicou uma taxa de quebra de $31 \%$ de endopróteses metálicas expansíveis, quando usadas em estenoses benignas, enquanto a taxa publicada por Nakajima et al. foi de $27 \%$, sendo que $18 \%$ apresentaram hemoptise letal.

As versões cobertas desse tipo de prótese visam eliminar o crescimento de tecido através dela, apesar do problema persistir nas áreas de metal não-coberto existentes nas pontas que possibilitam a fixação da mesma ${ }^{49}$. Este tipo de endoprótese tem sido utilizado para evitar a re-estenose devida ao crescimento de tecido de granulação ou neoplásico, e está relacionado a um aumento da taxa de manutenção da luz traqueobrônquica em longo prazo ${ }^{50-52}$.

Algumas recomendações ${ }^{53}$ foram publicadas para tentar evitar a ocorrência das complicações no caso das endopróteses metálicas: 1 - Ela deve ter seu diâmetro ajustado em relação às áreas não-afetadas proximal e distalmente à estenose. Isso minimiza erros na relação entre os diâmetros da via aérea e da endoprótese, o que pode levar à formação excessiva de tecido de granulação nas suas terminações e à falha na epitelização. Uma endoprótese muito estreita corre o risco de migrar e de perder o contato com a parede traqueal, não sendo incorporada à mucosa. 
Uma muito larga pode provocar necrose mural e distensão excessiva, o que pode levar à obstrução distal da traquéia; 2 - O comprimento da endoprótese deve ser calculado para ter $10 \mathrm{~mm}$ a mais do que a área comprometida em ambas terminações. Isso diminui a incidência de re-estenose nas extremidades, pois estas ficam sobre tecido normal; 3 - Um tratamento prolongado com "muco-reguladores" e esteróides inalatórios, bem como broncoscopias de controle parecem ser necessários.

\section{Indicações específicas}

O principal fator que influencia nos resultados do tratamento com próteses traqueais é a seleção correta dos pacientes. Devido as suas diferentes características, vantagens e complicações, os diversos tipos de próteses recebem indicações específicas, contudo estas não são absolutas sendo que, em alguns casos, observa-se o uso tanto de um tipo quanto de outro.

A escolha da prótese ideal esbarra, inicialmente, em uma diferença de fundamental importância entre elas: o método de colocação, principalmente o tipo de anestesia necessário. Enquanto próteses de silicone exigem o uso de broncoscopia rígida e anestesia geral, os modelos de metal auto-expansíveis permitem a colocação através de broncoscopio flexível, utilizando anestesia local. Assim, o primeiro aspecto a ser levado em conta é o estado pré-operatório do paciente e sua capacidade de suportar anestesia. Também deve ser levada em conta a experiência da equipe, uma vez que a broncoscopia rígida é mais trabalhosa e exige técnica mais apurada.

A estenose traqueobrônquica adquirida pode ser dividida em benigna e maligna. Entende-se a primeira como qualquer causa de estenose (conhecida ou não) de causa não maligna ${ }^{37}$. A possibilidade de ocorrer fadiga do material contra-indica o uso de prótese de metal expansível nesta categoria de estenose, pois ela pode ficar submetida a forças de estresse mecânico durante muito anos, comprometendo sua integridade ${ }^{47}$; sendo reportada uma taxa ${ }^{20}$ de $15 \%$ desta complicação. Endopróteses metálicas expansíveis devem ser evitadas em estenoses benignas também porque podem produzir dano à mucosa das vias aéreas após um longo de permanência $8,54,55$.

O FDA (Food and Drug Adminstration) americano, após revisão da literatura e recebimento de denúncias contra dispositivos médicos, advertiu ${ }^{56}$ contra o uso de próteses metálicas, de todas as marcas e de modelo coberto e não-coberto, nos casos de estenose benigna de traquéia devido à uma maior taxa de com- plicações relacionadas ao uso deste dispositivo nestes casos e à dificuldade de sua remoção, o que pode esgotar suas opções terapêuticas; sendo que o uso deste tipo de endoprótese só deve ser utilizado nos casos em que todas as outras opções de tratamento (inclusive a colocação de endopróteses de silicone) já tenham sido completamente exploradas. O FDA adverte também sobre a necessidade da colocação e remoção das endopróteses serem feitas por médicos treinados ou experientes e sobre a importância de uma seleção cuidadosa de pacientes.

Por serem mais duráveis e, principalmente por serem mais facilmente removidas e reposicionadas, as próteses de silicone rígido são mais adequadas, pois podem "acompanhar" a evolução da doença, sendo adaptadas de acordo com a necessidade. Ocasionalmente, a permanência de uma endoprótese na luz traqueal pode levar à estabilização do problema, o que permite a retirada definitiva da mesma ${ }^{23,39,57}$.

Assim, as próteses de silicone rígido são consideradas o padrão ouro para o tratamento da estenose benigna ${ }^{39,42,58}$, com o único revés de que a broncoscopia rígida necessária para a sua colocação é uma técnica difícil e pouco difundida entre os especialistas. Uma pesquisa ${ }^{59}$ publicada pelo American College of Chest Physicians, demonstrou que apenas 6\% dos pneumologistas americanos são capazes de realizar a broncoscopia rígida.

Para a estenose maligna, a colocação de próteses traqueais constitui o único tratamento imediato para compressão extra-luminal causada por tumores irresecáveis, estabilizando a via aérea enquanto o tumor primário é tratado com quimio ou radioterapia ${ }^{60,61}$. Não há uma orientação clara a respeito de que tipo de prótese deve ser usado, porém a redução dos sintomas na adoção desta medida paliativa para pacientes com doenças em estágio terminal já foram comprovados ${ }^{62}$.

As próteses metálicas são utilizadas quando existe uma dificuldade em assentar próteses de silicone devido à anatomia do local da lesão, quando é impossível colocar uma prótese de silicone larga o suficiente ou quando a compressão extrínseca é tanta que resulta em deformação da prótese de silicone ${ }^{22,37}$. Entretanto, questões ${ }^{62,63}$ têm sido levantadas questionando os reais benefícios de se prolongar a vida em pacientes cujo prognóstico e, principalmente, a qualidade de vida são tão ruins.

\section{CONCLUSÃO}

A Tabela 2 mostra um resumo dos trabalhos mais relevantes publicados sobre o uso de endopróteses em estenoses traqueobrônquicas. Como podemos observar as próteses metálicas apresentam menores 
taxas de migração e maiores taxas de complicações relacionadas ao seu uso por períodos mais longos, como formação de tecido de granulação e quebra. Portanto, reafirmando o que foi citado previamente deve ser a prótese de escolha para pacientes com estenoses neoplásicas cuja expectativa de vida não é longa. Esses casos se beneficiam de uma prótese que possa ser posicionada do broncoscopia flexível e que não precise ser revisada. Por outro lado, as endopróteses de silicone apresentam maior taxa de migração, porém menor complicação de longo prazo, portanto deve ser reservada para aqueles que necessitam de prótese temporária ou que necessitarão desta por um longo período de tempo (e provavelmente revisões e trocas).

Em conclusão, a análise das taxas de incidência de complicações (Tabela 2), das vantagens e desvantagens do tipo de cada endoprótese e das experiências pessoais relatadas na literatura, levou-nos a propor o seguinte algoritmo quando lidando com estenoses traqueais.

Tabela 2. Extremos entre as taxas de complicações para endopróteses traqueobrônquicas publicadas na literatura

\begin{tabular}{lcc}
\hline Complicações & Silicone & Metálicas expansíveis \\
\hline Migração & $5,0^{(42)}-18,7^{(40)} \%$ & $2,0^{(20)}-4,8^{(46)^{*}} \%$ \\
Tecido de & & \\
granulação & $0,9^{(36)}-16,0^{(39)} \%$ & $7,0^{(20)}-43,0^{(64)} \%$ \\
Obstrução por & & - \\
secreção & $3,6^{(65)}-31,0^{(41)} \%$ & - \\
Infecção & $1,63^{(36)} \%$ & $5,4^{(66)}-15,9^{(46)} \%$ \\
Quebra & - & $7,7^{(64)}-15,0^{(20)}$ \\
\hline
\end{tabular}

*Todos os casos ocorreram quando se tentou a aplicação da endoprótese em lesões com estenose severa.

Obs: Só entraram nesta tabela trabalhos com número de pacientes maior do que 36

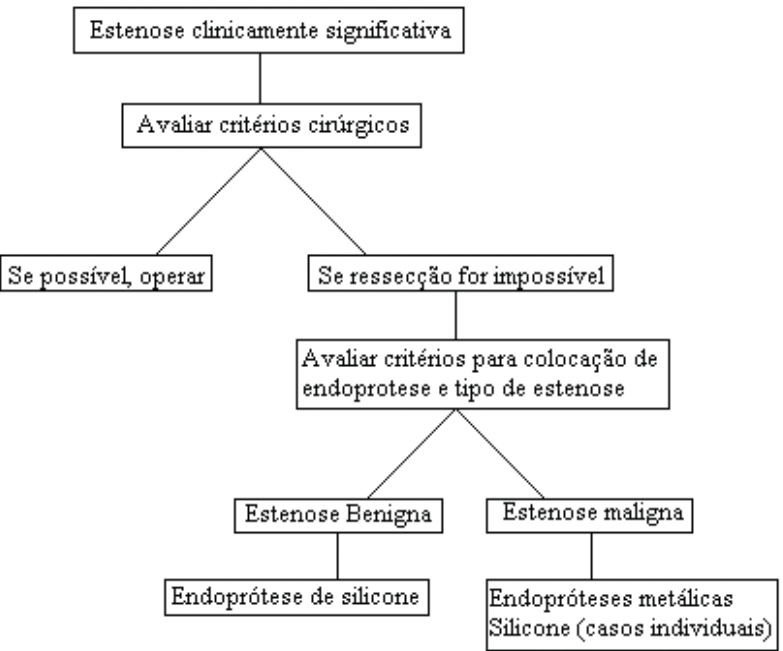

Gráfico 1. Algoritmo de conduta para estenoses clinicamente significativas Obs: Os critérios para colocação de endopróteses estão descritos na Tabela 3

\section{Tabela 3. Critérios para a colocação de endopróteses}

\section{Critérios $^{[43]}$ para a colocação de endopróteses}

Obstrução traqueobrônquica maligna, com compressão extrínseca para pacientes cujas opções de tratamento terapêutico já tenham sido exauridas.

Obstrução maligna persistente mesmo após ressecção broncoscópica e manobras de dilatação.

Pacientes com obstrução maligna que serão submetidos à radioterapia externa.

Estenose subglótica pós-intubação persistente após ressecção broncoscópica e tentativa de dilatação.

Estenose benigna complexa em pacientes que não são candidatos à cirurgia.

Estenose traqueal ou brônquica benigna causada por doenças inflamatórias ou infecciosas, enquanto aguarda-se a resposta ao tratamento sistêmico ou alguma pendência à cirurgia.

Traquebroncomalacia, extensa ou localizada independente da causa.

Estenose em local de anastomose após transplante pulmonar.

Fístulas traqueo ou broncoesofágicas. 
Anjos DM dos, Junqueira JJM, Gomes DBD, Terra RM, Minamoto H, Jatene FB. Tracheobronchial stents: terapeutic option for acquired tracheobronchial stenosis. Rev Med (São Paulo). 2007 jul.-set.;86(3):174-84.

\begin{abstract}
Tracheobronchial stenosis curses with dyspnea, stridor and obstructive pneumonia, and can be caused by tumors, extrinsic compression and tracheobronchomalacia, but the most frequent cause is the sequel resulting of prolonged intubation, occurring in about $1 \%$ intubations. The treatment of choice for such condition is resection of the compromised segment; however this procedure isn't possible in every patient and this led to the development of endoscopic palliative technique. Tracheobronchial stentintg is between one of such techniques that obtain the best results. Tracheobronchial stents can be divided manly between silicone tubes and self-expandable metallic stents, each one with specific advantages and disadvantages. The present study presents the main stent models, comparing their advantages e disadvantages and complications rates, aiming to systematize the ideal indications for every type of stent.
\end{abstract}

KEY WORDS: Constriction pathologic, Trachea/surgery. Prosthesis implantation. Dyspnea/ etiology.

\title{
REFERÊNCIAS
}

1. Law HJ, Barnhart K, Rowlett W, et al. Increased frequency of obstructive airway abnormalities with long-term tracheostomy. Chest. 1993;104:136-8.

2. McCulloch TM, Bishop MJ. Complications of translaryngeal intubation. Clin Chest Med. 1991;12:501-21.

3. Hoasjoe DK, Franklin SW, Aarstad RF, et al. Posterior glottic stenosis mechanism and surgical management. Laryngoscope. 1997;107:675-9.

4. Marel M, Pekarek Z, Spasova I, Pafko P, Schutzner $\mathrm{J}$, Betka $\mathrm{J}$, et al. Management of benign stenoses of the large airways in the university hospital in Prague, Czech Republic, in 1998-2003. Respiration. 2005;72(6):622-8.

5. Dutau H. Tracheal stenoses endoscopic treatment. Proceedings of the 12th World Congress for Bronchology, Boston, 2002. Bologna: Monduzzi Editore, 2002. p. 83-8.

6. Hanna E, Eliachar I. Endoscopically introduced expandable stents in laryngotracheal stenosis: the jury is still out. Otolaryngol Head Neck Surg. 116:97-103.

7. Wood DE. Tracheal tumors. In: Wood DE, Thomas CR, editors. Mediastinal tumors. Berlin: Springer-Verlag; 1995. p.87-93.

8. Nashef SAM, Dromer C, Velly JF, Labrousse L, Couraud L. Expanding wire stents in benign tracheobronchial disease: indications and complications. Ann Thorac Surg. 1992;54:937,940.

9. Mandour M, Remacle M, Heyning PV, Elwany S, Tantawy A, Gaafar A. Chronic subglottic and tracheal stenosis: endoscopic management vs. surgical reconstruction. Eur Arch Otorhinolaryngol. 2003;260:374,380.

10. Gaissert HA, Grillo HC, Wright CD, Donahue DM, Wain JC, Mathisen DJ. Complication of benign tracheobronchial strictures by self-expanding metal stents. J Thorac Cardiovasc. Surg. 2003;126:744-7.
11. Grillo HC, Donahue DM, Mathisen DJ, et al. Postintubulation tracheal stenosis: treatment and results. J Thorac Cardiovasc Surg. 1995;109:486-93.

12. Freitag L. Tracheobronchial stents. Eur Respir Mon. 1998;9:79-105.

13. Maddaus M, Pearson FG. Postintubation injury. In: Pearson FG, editor. Thoracic surgery. New York: Churchill Livingstone; 1995. p.251-65.

14. Wood DE. Airway stenting. Chest Surg Clin N Am. 2001;11(4):841-60.

15. Muller MR, Klepetko W, Rogy M, et al: Ergebnisse mit der queren Trachealresektion bei PostintubationsTrachealstenosen. Chirurg. 1991;62:547-51.

16. Brunings W, Albrecht W. Kirekte Endoskopie der Luft und Speisewege. Stuttgart: Enke; 1915. p.134-8.

17. Dumon JF. A dedicated tracheobronchial stent. Chest. 1990;97(2):328-32.

18. Wallace MJ, Charnsangabvej C, Ogawa K, Carrasco $\mathrm{CH}$, Wright KC, McKenna R, et al. Tracheobronchial tree: expandable metallic stents used in experimental and clinical applications (work in progress). Radiology. 1986:158:309-12.

19. Korpela A, Aarnio $P$, Sariola $H$, et al. Bioabsorbable self-reinforced poly-I-lactide, metallic, and silicone stents in experimental tracheal stenosis. Chest. 1999;115:490-5.

20. Shin JH, Song HY, Shim TS. Management of Tracheobronchial Strictures. Cardiovasc Intervent Radiol. 2004;27:314-24.

21. Montgomery WW. Silicone Tracheal T-tube. Ann Otol Rhinol Laryngol. 1974;83:71-5.

22. Grillo HC. Tracheal T tubes. Surgery of the trachea and bronchi. Cap 35.

23. Cooper JD, Pearson FG, Patterson GA, et al. Use of silicone stents in the management of airway problems. Ann Thorac Surg. 1984;47:371-8. 
24. Nomori $\mathrm{H}$, Horio $\mathrm{H}$, Suemasu K. Dumon stent placement via endotracheal tube. Chest. 1999;115(2):582-3.

25. Vinnograd I, Klin B, Brosh T, et al. A new intratracheal stent made from nitinol, an alloy with "shape memory effect”. J Thorac Cardiovasc Surg. 1994;107:1255-66.

26. Sesterhenn AM, Wagner HJ, Alfke H, Werner JA, Lippert BM. Treatment of benign tracheal stenosis utilizing selfexpanding nitinol stents. Cardiovasc Intervent Radiol. 2004;27(4):355-60.

27. Thornton MA, Rowley $\mathrm{H}$, Timon $\mathrm{C}$. A modified technique for nitinol stent insertion in the tracheobronchial tree. Otolaryngol Head Neck Surg. 2003;128(6):802-4.

28. Zannini P, Melloni G, Chiesa G, Carretta A. Selfexpanding stents in the treatment of tracheobronchial obstruction. Chest. 1994;106:86-90.

29. Wassermann K, Koch A, Muller-Ehmsen J, Reuter M, Michel O, Eckel HE. Clinical and laboratory evaluation of a new thin-walled self-expanding tracheobronchial silicone stent: progress and pitfalls. J Thorac Cardiovasc Surg. 1997;114(4):527-34.

30. Freitag L, Tekolf E, Greschuchna D. Development of a new insertion technique and a new device for the placement of bifurcated airway stents. Surg Endosc. 1994;8(12):1409-11.

31. Jacobs JP, Quintessena JA, Botero LM, et al. The role of airway stents in the management of pediatric tracheal, carinal, and bronchial disease. Eur J Cardiothorac Surg. 2000;18:505-12.

32. Sommer D, Forte V. Advances in the management of major airway collapse. Otolaryngol Clin North Am. 2000;33:163-77.

33. Mehta AC, Dasgupta A. Airway stents. Clin Chest Med. 1999;20:139-51.

34. Dasgupta A, Mehta AC. Use of Wallstents in central airway obstruction. Oper Techn Otolaryngol Head Neck Surg. 1999;10:264-70.

35. Vonk-Noordegraaf A, Postmus PE, Sutedja TG. Tracheobronchial stenting in the terminal care of cancer patients with central airways obstruction. Chest. 2001;120:1811-4.

36. Cavaliere S, Venuta F, Foccoli P, Toninelli C, La Face B. Endoscopic treatment of malignant airway obstructions in 2.008 patients. Chest. 1996;110(6):1536-42.

37. Saito $Y$, Imamura H. Airway stenting. Surg Today. 2005;35:265-70.

38. Vergnon JM, Costes F, Polio JCH: Efficacy and tolerance of a new silicone stent for the treatment of benign tracheal stenosis. Chest. 2000;118:422-6.

39. Martinez-Ballarin JI, Diaz-Jiménez JP, Castro MJ, Moya JA. Silicone stents in the management of benign tracheobronchial stenoses: tolerance and early results in 63 patients. Chest. 1996;109:626-9.

40. Puma F, Ragusa M, Avenia N, Urbani M, Droghetti A, Daddi $\mathrm{N}$, et al. The role of silicone stents in the treatment of cicatricial tracheal stenoses. J Thorac Cardiovasc Surg 2000;120:1064-9.

41. Schmidt B, Olze H, Borges AC. Endotracheal balloon dilatation and stent implantation in benign stenoses. Ann Thorac Surg. 2001;71:1630-4.
42. Wood DE, Liu YH, Vallie E, Karmy-Jones R, Mulligan MS. Airway stenting for malignant and benign tracheobronchial stenosis. Ann Thorac Surg. 2003;76:167-74.

43. Colt HG, Dumon JF. Airway stents: present and future. Clin Chest Med. 1995;16:465-78.

44. Xu X, Tajima $\mathrm{H}$, Ishioh $M$, Watari J, Miyashita $T$, Kumazaki $T$, et al. Study on the treatment of tracheobronchial stenosis using expandable metallic stents. J Nippon Med Sch. 2001;68(4).

45. Zakaluzny SA, Lane JD, Mair EA. Complications of tracheobronchial airway stents. Otolaryngol Head Neck Surg. 2003;128:478-88.

46. Saad CP, Murthy S, Kyizmanich G. Self-expandable metallic airway stents and flexible bronchoscopy: longterm outcomes analysis. Chest. 2003;124:1993-9.

47. Jantz MA, Silvestri GA. Silicone stents versus metal stents for management of benign tracheobronchial disease. Pro: metal stents. J Bronchol. 2000;7:177-83.

48. Rousseau H, Dahan M, Lauque D, Carre P, Didier A, Bilbao I, et al. Self-expandable prostheses in the trachealbronchial tree. Radiology. 1993;188:199-203.

49. Rodriguez AN, Diaz-Jimenez JP, Edell ES. Silicone stents versus metal stents for management of benign tracheobronchial disease: Con: Metal stents. J Bronchol. 2000;7:184-7.

50. Nomori H, Kobayashi R, Kodera K, Morinaga S, Ogawa K. Indications for an expandable metallic stent for tracheobronchial stenosis. Ann Thorac Surg. 1993;56:1324-8.

51. Kishi K, Kobayashi H, Suruda T, Ohata M, Sonomura T, Nishida N, et al. Treatment of malignant tracheobronchial stenosis by Dracon mesh-covered Z-stents. Cardiovasc Intervent Radiol. 1994;17:33-5.

52. Miyayama S, Matsui O, Kamimura R, Kakuta K, Takashima T. Partially covered Gianturco stent for tracheobronchial stricture caused by intraluminal tumor. Cardiovasc Intervent Radiol. 1997; 20:60-2.

53. Mostafa BE. Endoluminal stenting for tracheal stenosis. Eur Arch Otorhinolaryngol. 2003;260:465, 8.

54. Edell ES, Colt HG, Dumon JF. Tracheobronchial prostheses. In: Prakash UBS, editor. Bronchoscopy. New York: Raven Press; 1994. p.301-11.

55. Hramiec JE, Haasler GB. Tracheal wire stent complications inmalacia: implications of position and design. Ann Thorac Surg. 1997;63:209-13.

56. Minamoto H, Terra RM, Sancho LMM. Complicações das endopróteses metálicas de traquéia nos pacientes com doenças benignas das vias aéreas: notificação em saúde pública do FDA. Bol Pneumol Paulista. 2006; (31).

57. Bisson A, Bonnette P, Ben El Kadi N, Leroy M, Colchen A, Personne $\mathrm{C}$, et al. Tracheal sleeve resection for iatrogenic stenoses (subglottic, laryngeal, and tracheal). J Thorac Cardiovasc Surg. 1992;104:882-7.

58. Dumon JF, Cavaliere S, Diaz-Jimenez JP, et al. Seven years experience with the Dumon prosthesis. J Bronchol. 1996;3:6-10.

59. Colt HG, Prakash UB, Offord KP. Bronchoscopy in North America: survey by the American Association for Bronchology, 1999. J Bronchol. 2000;7:8-25. 
60. Kim H. Stenting therapy for stenosing airway disease. Respirology. 1998;3:221-8.

61. Shin JH, Kim SW, Shim TS, Jung GS, Kim TH, Ko GY, et al. Malignant tracheobronchial strictures: palliation with covered retrievable expandable nitinol stent. J Vasc Interv Radiol. 2003;14:1525-34.

62. Sutedja G, Schramel F, van Kralingen K, et al. Stent placement is justifiable in end stage patients with malignant airway tumor. Respiration. 1995;62:148-50.

63. Sheldon T. Netherlands published plan for euthanasia law [letter]. BMJ. 1999;319:467.
64. Noppen M, Stratakos G, D'Haese J, Meysman M, Vinken W. Removal of covered self-expandable metallic airway stents in benign disorders: indications, technique, and outcomes. Chest 2005;127;482-487

65. Dumon MC, Dumon JF, Perrin C, Blaive B. Silicone tracheobronchial endoprosthesis. Rev Mal Respir. 1999;16(4 Pt 2):641-51.

66. Dasgupta A, Dolmatch BL, Abi-Saleh WJ, Mathur PN, Mehta AC. Self-expandable metallic airway stent insertion employing flexible bronchoscopy: preliminary results. Chest. 1998;114:106-9. 